\section{ECCOMAS}

\section{Proceedia}

COMPDYN 2019

$7^{\text {th }}$ ECCOMAS Thematic Conference on Computational Methods in Structural Dynamics and Earthquake Engineering M. Papadrakakis, M. Fragiadakis (eds.) Crete, Greece, 24-26 June 2019

\title{
DENOISING CORRUPTED STRUCTURAL VIBRATION RESPONSE: CRITICAL COMPARISON AND ASSESSMENT OF RELATED METHODS
}

\author{
Gabriele RAVIZZA $^{1}$, Rosalba FERRARI ${ }^{1}$, Egidio RIZZI ${ }^{*}$, \\ Vasilis DERTIMANIS ${ }^{2}$ and Eleni N. CHATZI ${ }^{2}$ \\ ${ }^{1}$ University of Bergamo, Department of Engineering and Applied Sciences, \\ viale G. Marconi 5, I-24044 Dalmine (BG), Italy \\ ${ }^{*}$ Corresponding Author, e-mail: egidio.rizzi@unibg.it \\ ${ }^{2}$ ETH Zürich, Institute of Structural Engineering, \\ Department of Civil, Environmental and Geomatic Engineering, \\ Stefano-Franscini-Platz 5, CH-8093 Zürich, Switzerland
}

\begin{abstract}
Vibration-based Structural Health Monitoring (SHM) is receiving increasing attention due to the high technological level that the developed methodologies are nowadays reaching, together with the increasing quest for the implementation of effective, but ideally of low-cost, monitoring instrumentation. The latter is relatively easy to deploy and allows for the recording of the structural vibration response at multiple locations, which, for large strategical infrastructures, such as high-rise buildings, bridges, wind farms, etc. may be of critical importance. On the other hand, low-cost instrumentation may typically be accompanied by high Noise-to-Signal (N/S) ratios, contaminating the structural response, increasing the induced uncertainties and rendering the implementation of SHM methods more difficult. This calls for appropriate and effective denoising processes. In tackling such an associated denoising problem, several methods have been proposed and are currently under further development. Among specific variants, the utilization of multi-rate filter banks, especially the one based on Discrete Wavelet Transform (DWT), as well as the application of Singular Value Decomposition (SVD), have revealed to be rather effective. Yet, they have mostly been applied to problems where structural vibration response signals originate from specific behavioral classes, as, e.g., in monitoring applications of rotating machinery. In this study, the aforementioned methods are reconsidered and reimplemented; then, assessed on noise-corrupted vibration response signals related to civil engineering applications. Different Noise-to-Signal (N/S) ratios and excitation types are considered, i.e. earthquake and ambient vibration input. Advantages and limitations of both denoising approaches are presented and discussed, with a critical comparison. The outcomes prove the effectiveness of the considered methods in clarifying earthquake response signals, yet also their difficulty in purifying ambient vibration signals, motivating further specific research on the filtering of this latter common class of structural response.
\end{abstract}

Keywords: Noise-corrupted signal; Noise-to-Signal (N/S) ratio; Denoising methods; Discrete Wavelet Transform (DWT); Singular Value Decomposition (SVD); Seismic response; Ambient vibration. 


\section{INTRODUCTION}

Due to ever increasing structural requirements and loading condition magnitudes to which civil engineering structures are continuously subjected, an effective and accurate evaluation of the safety of existing structures, as well as an appropriate estimation of their remaining lifetime, become more and more necessary. In particular, in the last few years, the increasing interest in predicting the potential deterioration and collapse of such critical infrastructures by collocating on them various sensors for detecting data related to their dynamic response, has led to the emergence of the field of Structural Health Monitoring (SHM). Many case studies in which the structural vibration response has been employed as a critical and directly measurable quantity for evaluating structural safety, may be found in the literature [1-4]. From all these examples, the availability of reliable data on structural safety, usability and fatigue resistance, represents an essential point for efficient SHM, aiming at preserving structural integrity over time. However, often either no updated information on the structural characteristics is available or it may result incomplete, or even noise affecting the signals may considerably alter the useful information embedded within the response signals themselves. Consequently, in many cases, the application of denoising techniques aiming at enhancing the data quality by removing the noise affecting the signals, might become necessary.

Several denoising approaches for dealing with signals have been developed and reported in the literature [5-7]. In particular, the application of band pass filters, such as high-pass and lowpass filters, may be considered as a most simple and rudimentary denoising method. The Fourier Transform might also be employed for denoising purposes [8]. However, all these mentioned approaches are ineffective when the noise is concentrated in a frequency band similar to that of the useful part of the signal, and this occurs in several real cases. In fact, this would mean removing not only the noise but also a relevant portion of the meaningful signal, which instead should be preserved.

In the last two decades, this has led to search for alternative denoising approaches able to overcome this issue, by removing the contaminated part of the signal without losing the important information contained within it. In particular, two important denoising approaches are identified, as focus herein, i.e., one based on a Discrete Wavelet Transform (DWT) implementation [9] and one based on a Signal Value Decomposition (SVD) algorithm [10]. In addition, a further interesting approach to the problem of signal denoising is the effectuated use of a Kalman Filter (KF), as a powerful tool to enhance noise-corrupted data, as presented in recent publications [11-13]. Specifically, in Ravizza et al. [13], a KF algorithm has been integrated into a Heterogeneous Data Fusion (HDF) scheme, aiming at improving the quality of displacement response signals detected on a numerical dynamic system.

In this paper, DWT- and SVD-based denoising approaches are reconsidered and independently implemented, in order to explore their effectiveness in dealing with signal typologies typical of civil engineering contexts, namely structural vibration response signals. This may open up to new perspectives in the post-processing of such signals; in fact, although these techniques have been already tested, for instance, on gravity and magnetic signals, biological signals such as electrocardiograms (ECG) or electroencephalograms (EEG), or even acoustic signals and pressure signals, their application on structural vibrational signals, typical of the civil engineering field, has not been deeply inspected yet. Furthermore, structural response data are crucial for SHM purposes, as their observation over time, also supported by the wide development of modal identification techniques for the acquisition of the structural modal features [14-16], may reveal variations in the physical properties of the monitored structure. This 
might be related to the possible appearance of damage and, consequently, it could lead to a decay of the structural performance characteristics.

In particular, within the present analysis, seismic response and ambient vibration signals, in terms of acceleration, will be considered. This choice is motivated by the fact that, since these signals display a very different nature, i.e., non-stationary (seismic excitation) vs. stationary (ambient vibration), they could bring out different aspects inherent to the process of denoising, depending on the performed approach. A ten-story shear-type building is assumed as a benchmark reference structure, in order to generate simulated response signals, which are then subsequently contaminated with different levels of noise, and further denoised by means of the proposed techniques.

The main goal which this study aims to achieve is twofold:

- to inspect the effectiveness of denoising methods in dealing with response signals which are typical of the civil engineering context, i.e., ambient vibration and seismic acceleration response signals, through the assessment of their performance based on the comparison between the reconstructed denoised signal and the original clean one;

- to shed light on strengths and limitations of DWT- and SVD-based denoising approaches, depending on the amount of noise that affects the data, and as referring to the type of the response signal (ambient vs. seismic).

The paper is organized as follows. In Section 2 a brief description of the analysis procedure is provided, through the presentation of the benchmark dynamical system, the synthetic generation of last-floor's acceleration response signals (considering both seismic excitation and ambient vibration), and the process of their distortion by adding a Gaussian white noise. In Section 3, DWT- and SVD-based denoising approaches are performed on data affected by increasing noise levels, and results are presented and commented on. A critical comparison based on the performance evaluation of the two considered techniques is also presented. Conclusions and global remarks are finally outlined in Section 4, together with some possible future research perspectives.

\section{ANALYSIS PROCEDURE}

\subsection{Problem statement}

The traditional formulation of a generic signal denoising problem may be expressed according to the following relation:

$$
\mathbf{y}=\mathbf{x}+\mathbf{n}
$$

where $\mathbf{y}=\left(y_{1}, \ldots, y_{i}, \ldots, y_{n}\right)$ is the noise-affected signal which has to be processed, and that may be interpreted as the sum of a clean (noise-free) signal $\mathbf{x}=\left(x_{1}, \ldots, x_{i}, \ldots, x_{n}\right)$, which embeds the useful information to be preserved, and an additive noise signal $\mathbf{n}=\sigma\left(n_{1}, \ldots, n_{i}, \ldots, n_{n}\right)$, of intensity given by standard deviation $\sigma$, which may be modeled as a stationary independent zero-mean Gaussian term [17,18], superimposed on the useful signal and altering its content.

The application of each numerical denoising technique aims at achieving a best approximation $\widehat{\mathbf{x}}=\left(\widehat{x}_{1}, \ldots, \widehat{x}_{i}, \ldots, \widehat{x}_{n}\right)$ of original (noise-free) signal $\mathbf{x}$, starting from noise-affected signal $\mathbf{y}$, removing most of the unwanted noise without losing the useful information. It is 
worth mentioning that, at least at this preliminary stage of research, all signals involved within the analyses are constituted by synthetic signals, since this assumption allows to more deeply explore all possible strengths and limitations of the two studied denoising methods, rather than and before eventually considering real signals.

Several approaches have been presented in the literature in order to assess the effectiveness of denoising techniques [19-21]. Here, since both original (clean) signal $\mathbf{x}$ and denoised signal $\widehat{\mathbf{x}}$ are available, the Percentage Root Mean Square Difference (PrmsD) [22] value can be employed toward such purposes:

$$
\operatorname{PrmsD}=100 \cdot \sqrt{\frac{\sum_{i=0}^{n}\left(x_{i}-\widehat{x}_{i}\right)^{2}}{\sum_{i=0}^{n} x_{i}^{2}}}
$$

Small values of PrmsD are desirable for achieving a reliable approximation of original signal $\mathbf{x}$. In this study, PmrsD is considered as an evaluation term to compare the performances of DWTand SVD-based denoising approaches in processing both stationary and non-stationary response signals.

\subsection{Description of benchmark structure}

In order to generate simulated acceleration response signals, a one-bay ten-story shear-type building is taken as a reference structure. For making the analysis as truthful as possible, realistic values of mass, stiffness and damping are assumed. In particular, for the first-floor, values of mass $m_{1}=100 \mathrm{t}$ and stiffness $k_{1}=4 \cdot 10^{6} \mathrm{kN} / \mathrm{m}$ are considered, while the upper floors are characterized by values of mass $m_{i}=80 \mathrm{t}$ and stiffness $k_{i}=3 \cdot 10^{6} \mathrm{kN} / \mathrm{m}$, with $i=2, \ldots, 10$. Furthermore, a modal damping ratio $\zeta_{i}=5 \%$, as typical of reinforced concrete structures, is assumed, for all the modes.

During the analysis, two different input typologies are considered acting on the structure, i.e. an earthquake acceleration excitation, acting at the base, and an ambient vibration force distribution, applied along the structure with a linear variation (increasing from bottom to top of the building). In both cases, the last-floor acceleration response signal is solely monitored, as it shall be associated to the most critical response values and, consequently, it may be enough to well characterize the whole structural response of the analyzed building.

\subsection{Generation of noise-affected response signals}

In this section, the procedure of generation of noise-affected response signals is shown. In particular, a zero-mean Gaussian white noise is added to the original (noise-free) signals, aims at simulating the intrinsic error characterizing the sensor technology, as well as the errors which might occur "in situ" at the signal acquisition stage. The fact that the added Gaussian white noise affects each single frequency component indifferently over the whole length of the signal, makes its removal always a challenging task. Here, the Noise-to-Signal (N/S) ratio is used to quantify the amount of noise, in relation to the useful signal. Although in today's sensor technology N/S ratios greater then $20 \%$ might be considered as unrealistic, or extremely rare, for a complete understanding of the limitations of the explored denoising techniques, in the present analysis N/S ratios up to 50\% are taken into account. Two different types of excitation 
are considered as acting on the benchmark structure, i.e., seismic input and ambient vibration input; whereupon, performing a classic direct analysis, the dynamic response of the system is obtained.

The analyzed non-stationary input is the earthquake excitation that struck Kalamata (Greece) in 1986, characterized by a Peak Ground Acceleration (PGA) of $0.24 \mathrm{~g}$, magnitude of $M=5.9$ and focal depth of $22 \mathrm{~km}$. Such a seismic excitation has been assumed acting at the base of the reference building. The numerically-determined acceleration response signal related to the tenth floor is first obtained and then contaminated by adding a white Gaussian noise level of $25 \%$ N/S ratio, as depicted in following Fig. 1.
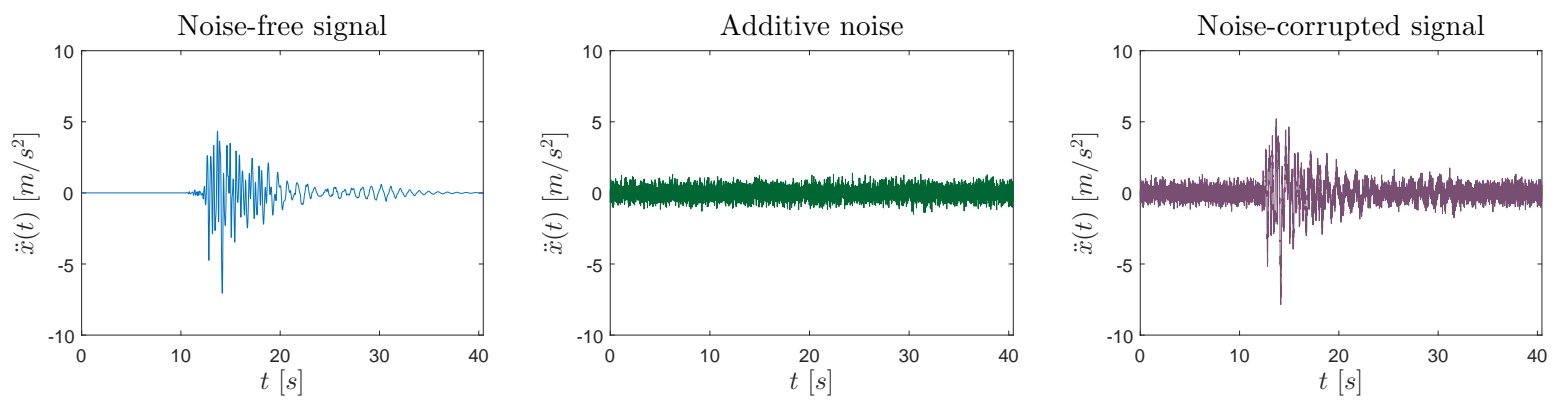

Figure 1: 10th-floor acceleration response signal of the benchmark structure under earthquake excitation input and subsequent noise addition process $(25 \% \mathrm{~N} / \mathrm{S}$ ratio $)$.

Ambient vibration, instead, represents the stationary excitation to which the structure is subjected to during its regular operating condition, and is commonly associated to environmental loads like wind, traffic load or wave motion. The last-floor dynamic acceleration response of the benchmark structural system under ambient vibration input is represented in Fig. 2, as well as the process of data corruption through the addition of a Gaussian white noise characterized by a $\mathrm{N} / \mathrm{S}$ ratio of $25 \%$.
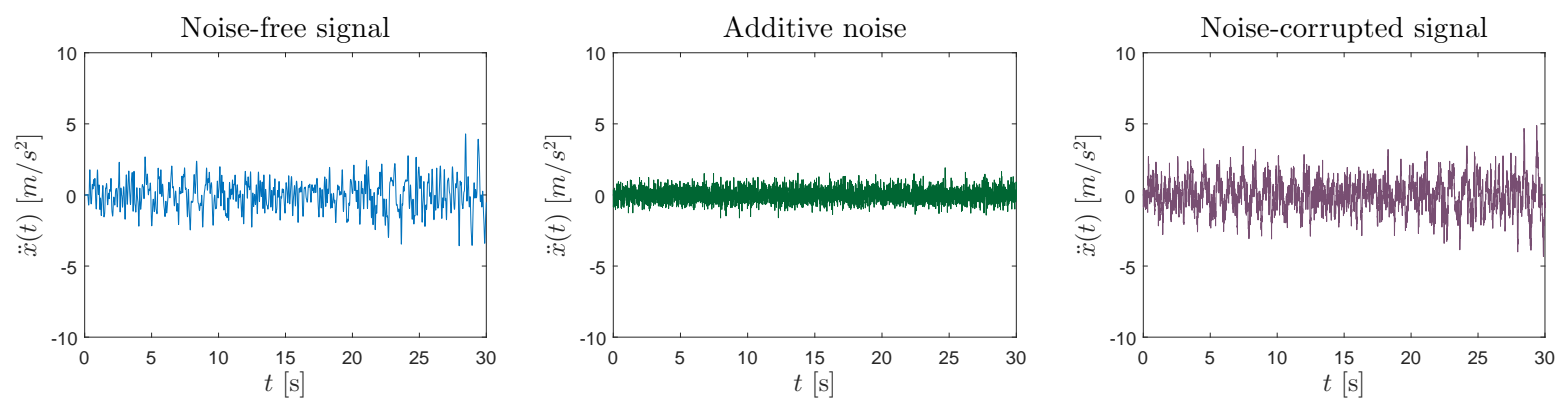

Figure 2: 10th-floor acceleration response signal of the benchmark structure under ambient vibration input and subsequent noise addition process (25\% N/S ratio).

The so generated noise-corrupted signals are now processed within a denoising implementation based on the application of DWT and SVD. The outcomes of the analysis are presented in next Section 3. 


\section{ANALYSIS RESULTS}

\subsection{DWT-based denoising application}

The optimal calibration of the DWT-based denoising approach for dealing with seismic response signals has been first investigated through a criterion of minimization of the PmrsD index between the original (noise-free) signal and the denoised one. For this purposes, several mother wavelets (i.e. Symlet, Coifet, Daubechies, Biorthogonal, Reverse Biorthogonal and Discrete Meyer) and thresholding rules (i.e. Heuristic SURE, Sqtwolog, Minimax and Rigorous SURE) have been explored, with the optimal solution being identified in the adoption of Smylet with two oscillations in its mother wavelet combined with Heursure hard thresholding, at decomposition level 2. The results of the DWT-based denoising performed on a seismic acceleration response signal corrupted with a $25 \%$ N/S ratio are shown in Fig. 3. The small error which may be appreciated between the original signal and the denoised one reveals the effectiveness of the method in the clarification of such a non-stationary signal.

A similar procedure has then been applied for the denoising of an ambient vibration response signal. However, even though the denoising method based on DWT has been suitably recalibrated for dealing with this specific class of signals, in this latter case, the error which occurs between the original signal and the denoised one becomes more visible, as observed in the last graph in Fig. 4. In particular, here, the combination of Coifet, featuring two oscillations in its mother wavelet and Minimax hard thresholding at decomposition level 3 is adopted. The difficulty in the denoising of stationary signals and, in general, the relevant discrepancy in the obtained results seems to clearly show how the typology of the processed signal strongly affects the performances of the DWT-based denoising technique.

\subsection{SVD-based denoising application}

The SVD-based implementation exploited in this study for denoising purposes is based on the computing of the singular values associated to so-called Hankel matrix $\mathbf{A}$, which contains the noise-corrupted signal components that ought to be denoised. Since it has been shown that the noise mainly affects the small singular values of the signal, it follows that by removing those lower than a selected threshold, a new matrix $\hat{\mathbf{A}}$ (of a lower rank) containing solely the denoised signal components, may be obtained. Thus, in this process, the critical point is represented by the choice of the threshold value. To this end, here, a criterion based on the minimization of the PrmsD index between the noise-free and denoised signal has been adopted, which has led to the selection of a threshold value of 10 .

In Fig. 5, the results of the SVD-based denoising approach performed on the non-stationary seismic response signal are shown. It may be asserted that, through the proposed SVD implementation, and despite the considered significant level of noise (i.e. $25 \% \mathrm{~N} / \mathrm{S}$ ratio), the original signal has been almost completely reconstructed, as demonstrated by the very small error that can be appreciated.

A similar analysis has then been performed on the stationary ambient vibration response signal and, contrary to what was seen for the denoising approach based on DWT, in this case, satisfactory outcomes may still be achieved, as represented in Fig. 6. Thus, the SVD-based denoising technique seems to better fit the different nature of the considered input, providing a more stable solution and, thus, leading to a more reliable estimate of the original reference signal. 

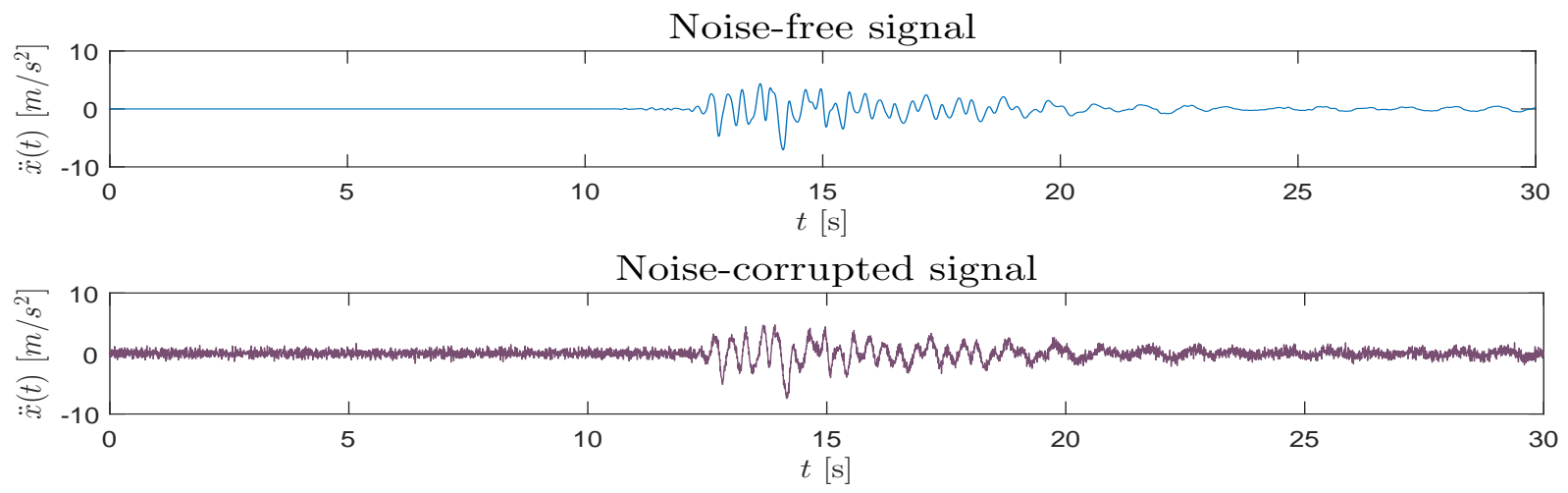

Denoised signal

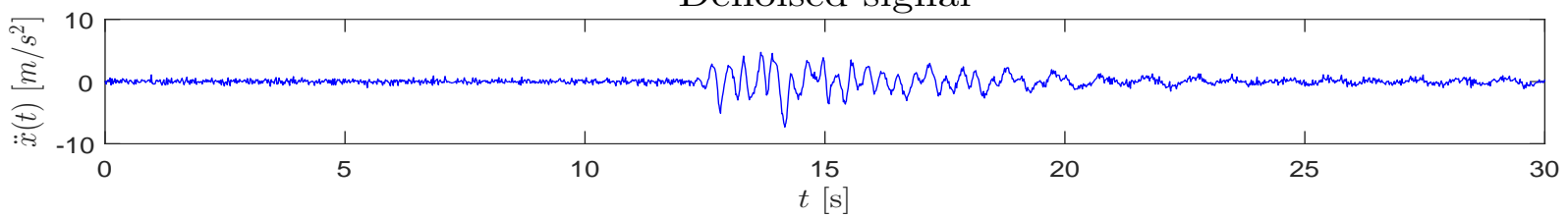

Error

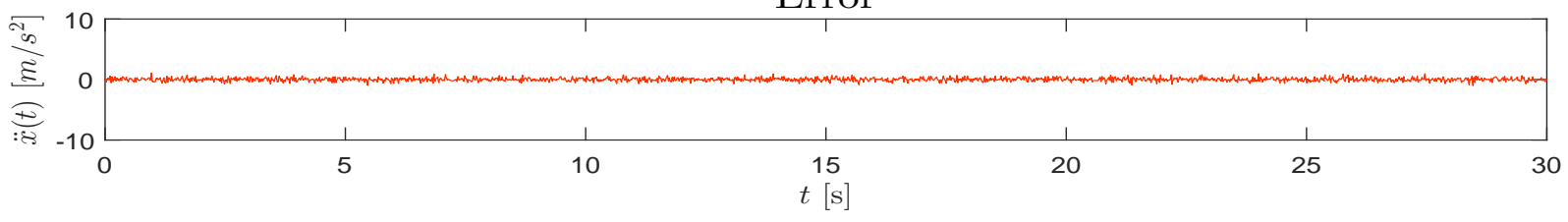

Figure 3: DWT-based denoising of a noise-corrupted $\ddot{x}(t)$ response signal under seismic input (25\% N/S ratio).

Noise-free signal

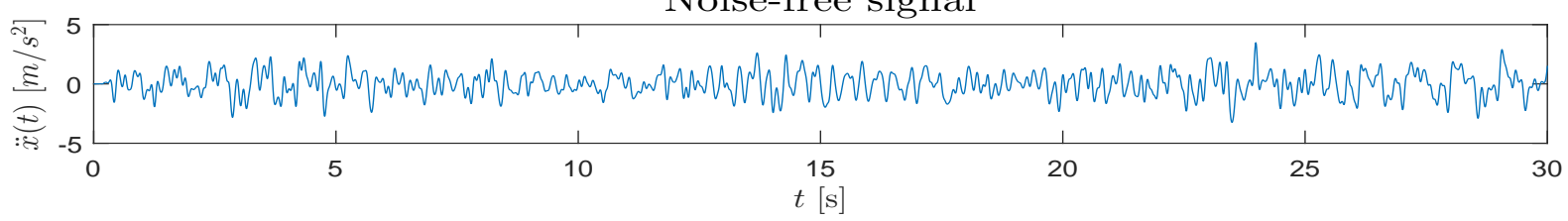

Noise-corrupted signal

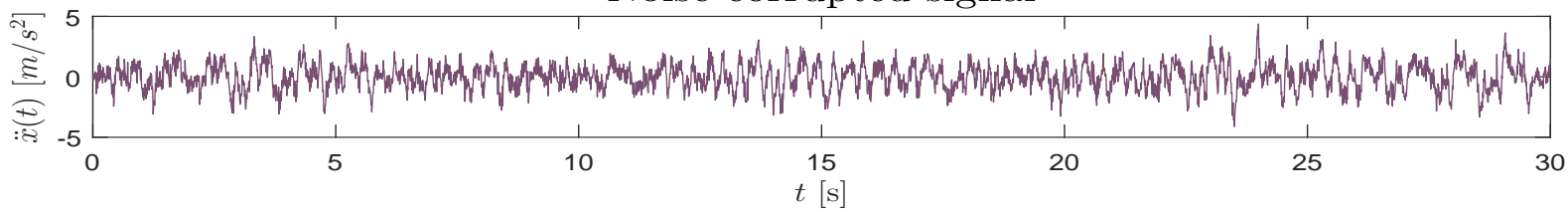

Denoised signal

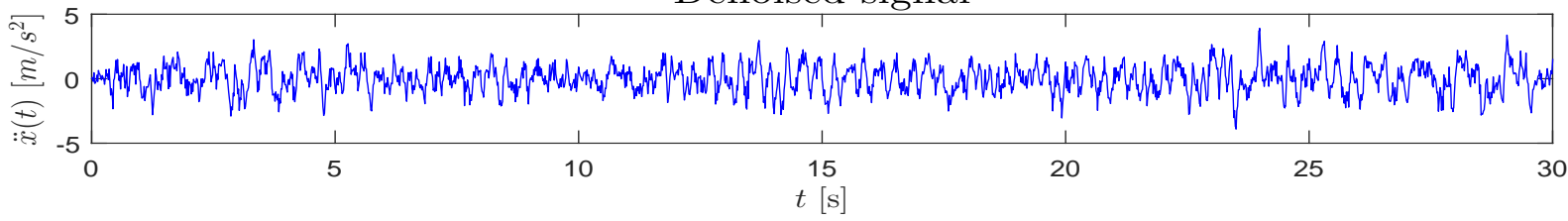

Error

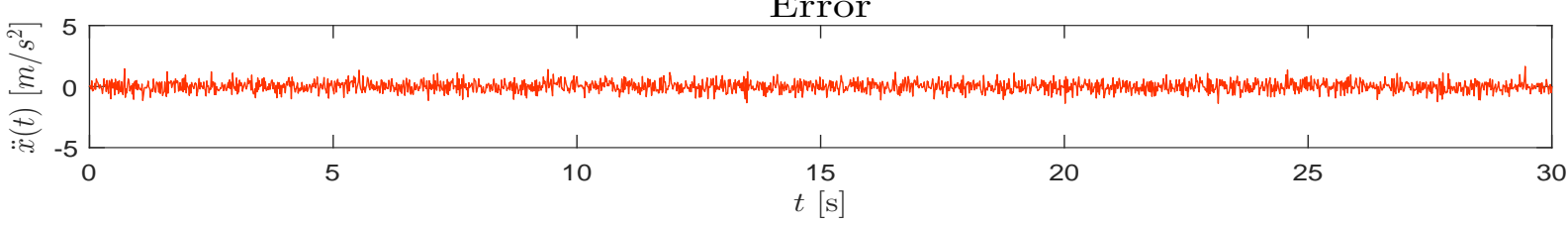

Figure 4: DWT-based denoising of noise-corrupted $\ddot{x}(t)$ response signal under ambient vibration (25\% N/S ratio). 


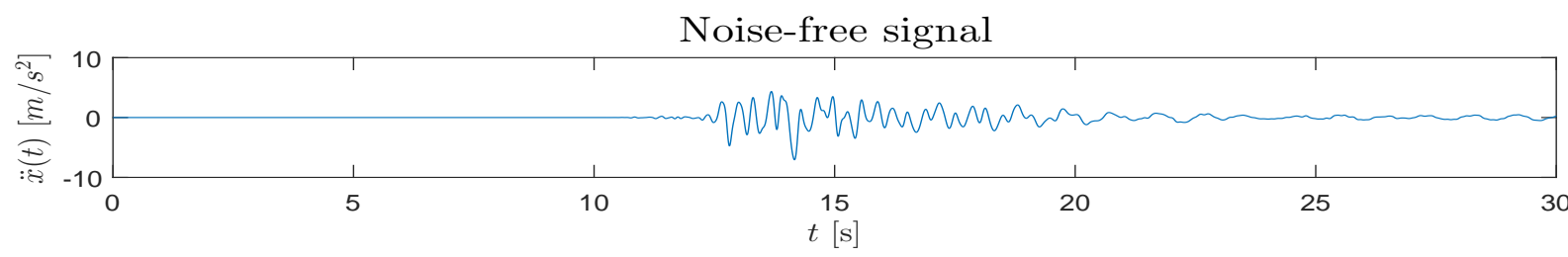

Noise-corrupted signal

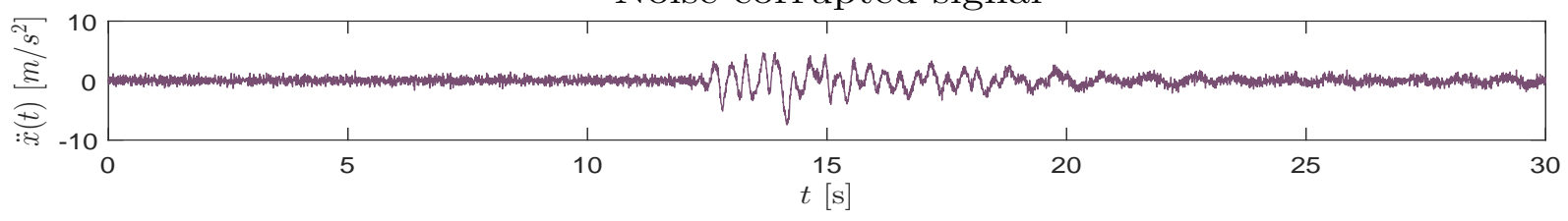

Denoised signal

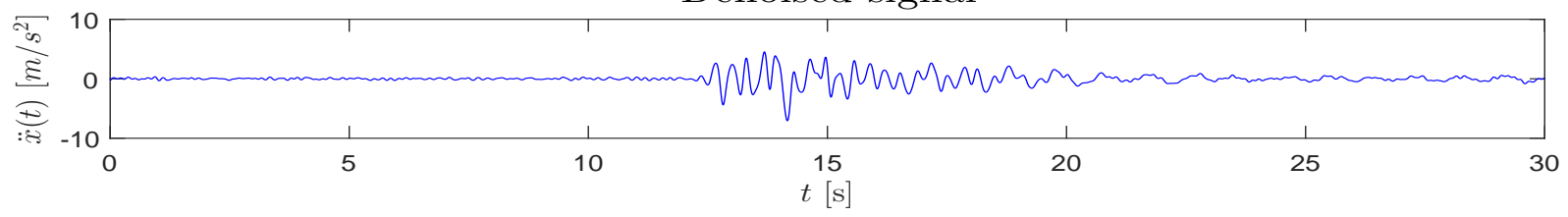

Error

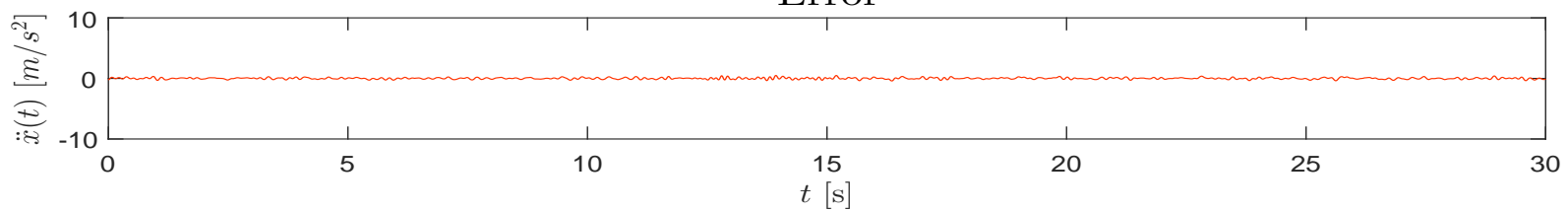

Figure 5: SVD-based denoising of a noise-corrupted $\ddot{x}(t)$ response signal under seismic input (25\% N/S ratio).

Noise-free signal

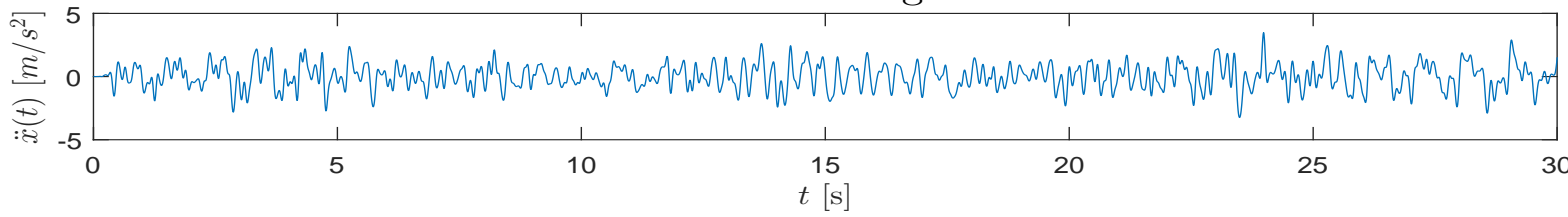

Noise-corrupted signal

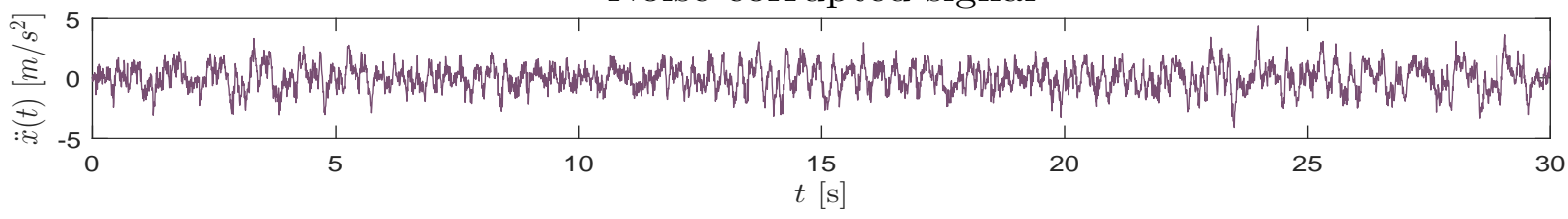

Denoised signal
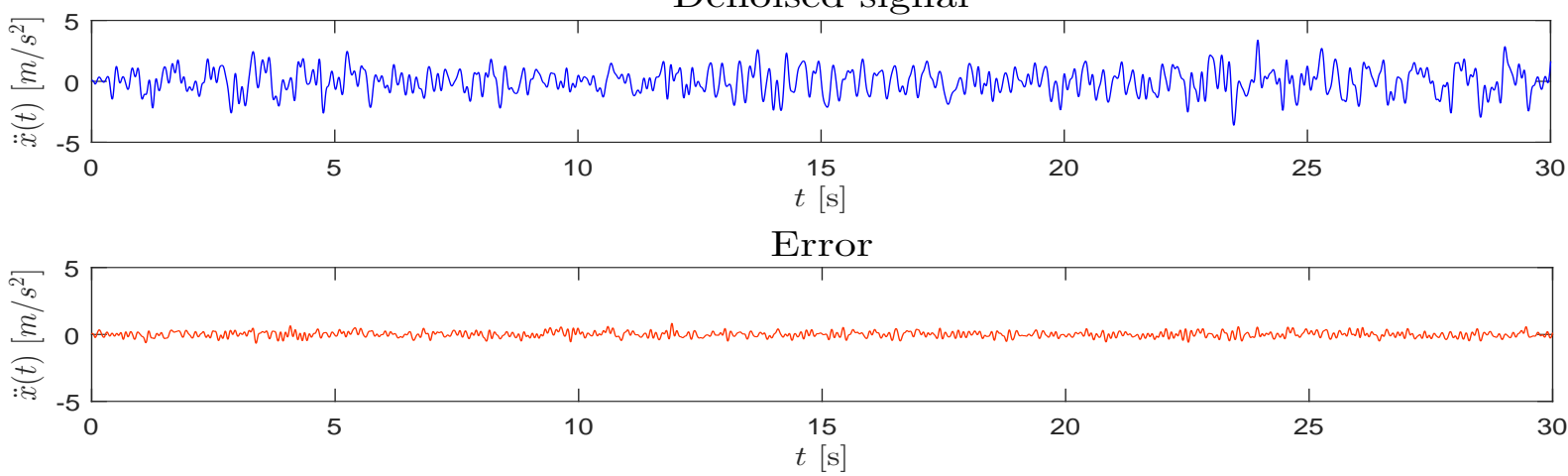

Figure 6: SVD-based denoising of a noise-corrupted $\ddot{x}(t)$ response signal under ambient vibration (25\% N/S ratio). 


\subsection{DWT- vs. SVD-based denoising techniques}

In this section, a critical comparison based on the performance evaluation of the two analyzed denoising approaches in clarifying noise-affected response signals is presented. The effectiveness of both methods is assessed, depending on the typology of the processed signal (non-stationary vs. stationary) and on the level of Gaussian white noise added to the source data. For academic purposes, N/S ratios up to $50 \%$ are considered within the analysis, and a PrmsD value between the reference signal and the denoised one is computed for each noise level. Results are summarized in following Table 1, and depicted in Fig. 7.

\begin{tabular}{|c|c|c|c|c|c|c|c|c|c|c|c|c|}
\hline $\mathrm{N} / \mathrm{S}$ ratio [\%] & & & 5 & 10 & 15 & 20 & 25 & 30 & 35 & 40 & 45 & 50 \\
\hline \multirow{4}{*}{ PrmsD [\%] } & \multirow{2}{*}{ Seismic excitation } & DWT & 0.06 & 0.13 & 0.18 & 0.20 & 0.88 & 1.18 & 1.23 & 1.39 & 2.11 & 2.69 \\
\hline & & SVD & 0.23 & 0.34 & 0.46 & 0.55 & 0.44 & 0.69 & 0.59 & 0.55 & 0.60 & 0.71 \\
\hline & \multirow{2}{*}{ Ambient vibration } & DWT & 0.59 & 1.25 & 2.64 & 3.80 & 4.58 & 4.93 & 5.23 & 5.90 & 6.21 & 6.82 \\
\hline & & SVD & 0.15 & 0.07 & 0.21 & 0.39 & 0.57 & 0.53 & 0.66 & 1.19 & 2.33 & 2.88 \\
\hline
\end{tabular}

Table 1: PrmsD [\%] index between original and denoised signal for different signal typologies and N/S ratios: DWT vs. SVD.

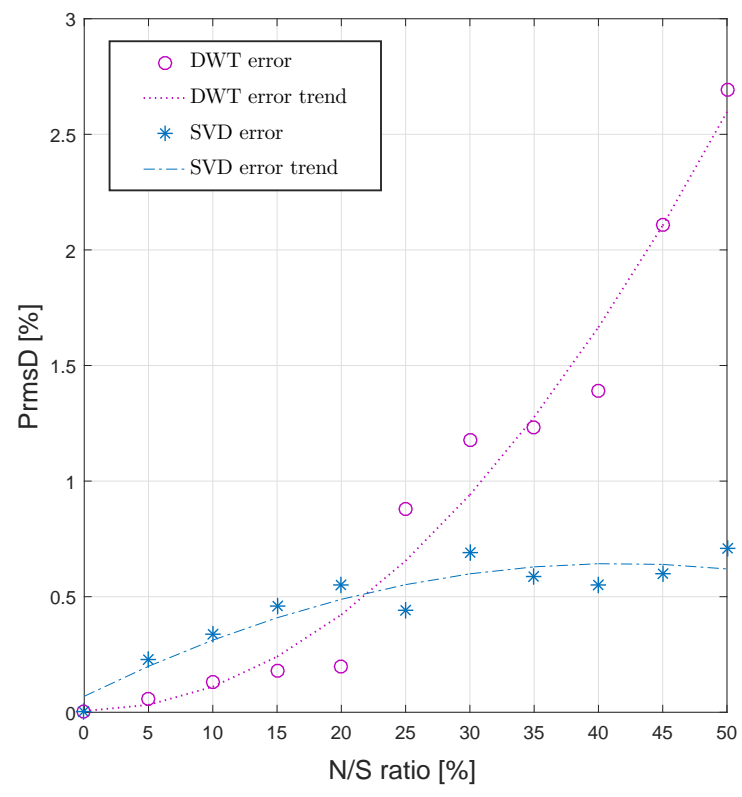

(a)

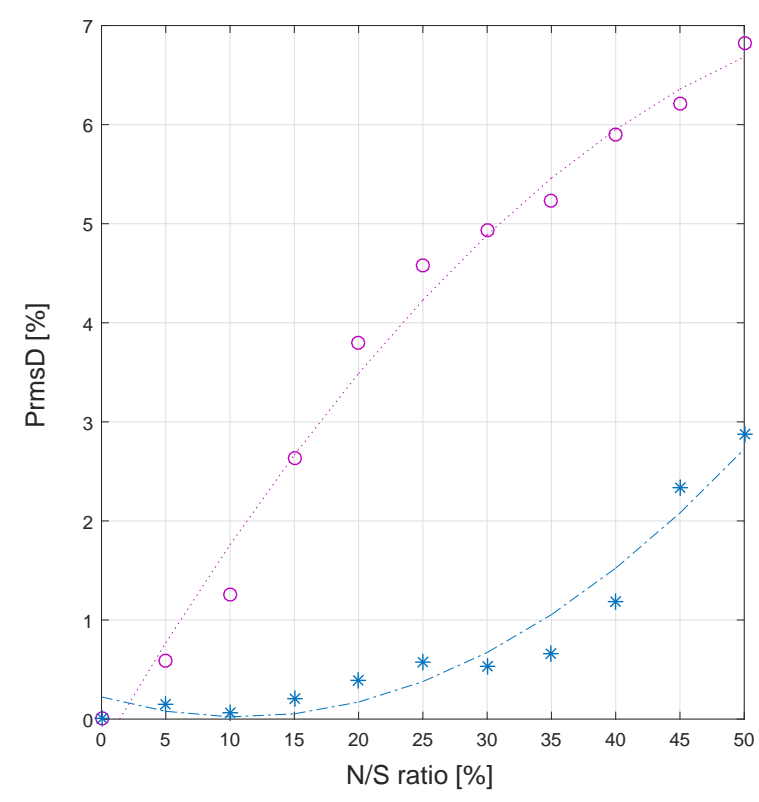

(b)

Figure 7: Effectiveness evaluation of DWT- and SVD-based denoising techniques for increasing N/S ratios and different signal typologies: (a) earthquake excitation; (b) ambient vibration. Notice that scales on the PrmsD axes are different, almost twice for $(b)$. Indicated trends come from a polynomial fit of degree 2.

From the obtained outcomes it may be deduced that both considered denoising approaches seem to be well suited for dealing with non-stationary signals, and in particular for seismic response. However, the analysis performed on ambient vibration response has shown that, for the denoising of stationary signals, only the SVD-based technique leads to reliable estimates. This technique seems less affected by the level of noise considered in the source signals, in comparison to the DWT-based approach. Its robustness may also be better appreciated by the 
graphs in Fig. 7, which plot the data in Table 1, where the error trends (in terms of PrmsD index) of the two denoising approaches are compared, considering the two studied typologies of source response signals.

\section{CONCLUSIONS}

In this paper, two different approaches for the denoising of (acceleration) structural response signals have been presented, i.e. one based on Discrete Wavelet Transform (DWT) and one based on Singular Value Decomposition (SVD). These two denoising techniques have been implemented with reference to simulated response signals typical of the civil engineering context, such as earthquake excitation and ambient vibration response signals. A ten-story shear-type building has been assumed as a benchmark structure, in order to generate the source noiseaffected signals, and the last-floor acceleration response has been monitored.

Salient research contributions which this study is bringing to light may be briefly summarized as follows:

- stationary signals proved more problematic to purify than non-stationary ones, due to their almost constant distribution in time and frequency;

- the effectiveness of the SVD-based denoising method has been fully proven. It can be stated that it constitutes a rather robust technique, which is able to return reliable estimates of the original (noise-free) signal, despite for the significant amount of additive noise assumed on the source data. Moreover, this technique seems to be suitable for both nonstationary and stationary signals;

- The DWT-based approach may be considered as useful for the denoising of non-stationary signals, such as seismic response signals, especially for N/S ratios lower than 20\%, where it may provide even better results than those for the SVD-based approach. However, the performed analysis has proven also its limitations in the processing of stationary signals, for which the application of the denoising method based on SVD is instead suggested.

Finally, after this first necessary phase of effectiveness assessment of the two denoising methods, in which only simulated signals have been considered, future developments shall concern the employment and denoising of real response signals (accelerations but also displacements), within the developed denoising procedure.

\section{Acknowledgments}

Public research support from "Fondi di Ricerca d'Ateneo ex 60\%" and a ministerial doctoral grant and funds at the ISA Doctoral School, University of Bergamo, Department of Engineering and Applied Sciences (Dalmine), are gratefully acknowledged.

\section{REFERENCES}

[1] Lee, J.J., Fukuda, Y., Shinozuka, M., Cho, S. and Yun, C.B. (2007), Development and application of a vision-based displacement measurement system for structural health monitoring of civil structures. Smart Structures and Systems, 3(3): 373-384.

[2] Ferrari, R., Froio, D., Chatzi, E.N., Gentile, C., Pioldi, F. and Rizzi, E. (2015), Experimental and numerical investigation for the structural characterization of a historic RC arch bridge. In: COMPDYN 2015, 5th ECCOMAS Thematic Conference on Computational Methods in Structural Dynamics and Earthquake Engineering, Crete Island, Greece, 25-27 May 2015, 1: 2337-2353, available online in Eccomas Proceedia, 
www.eccomasproceedia.org/conferences/thematic-conferences/compdyn-2015/3542.

[3] Ferrari, R., Pioldi, F., Rizzi, E., Gentile, C., Chatzi, E.N., Klis, R., Serantoni, E. and Wieser, A. (2015), Heterogeneous sensor fusion for reducing uncertainty in Structural Health Monitoring. In: UNCECOMP 2015, 1st ECCOMAS Thematic Conference on International Conference on Uncertainty Quantification in Computational Sciences and Engineering, Crete Island, Greece, 25-27 May 2015, pp. 511-528, available online in Eccomas Proceedia, www.eccomasproceedia.org/conferences/thematic-conferences/uncecomp$2015 / 4289$.

[4] Ferrari, R., Pioldi, F., Rizzi, E., Gentile, C., Chatzi, E.N., Serantoni, E. and Wieser, A. (2016), Fusion of wireless and non-contact technologies for the dynamic testing of a historic RC bridge. Measurement Science and Technology, 27(12): 1-19.

[5] Buades, A. and Coll, B. (2006), A review of image denoising algorithms, with a new one. Multiscale Modelling and Simulations, 4(2): 490-530.

[6] Chen, G. and Bui, T. (2003), Multiwavelets denoising using neighboring coefficients. IEEE Signal Processing Letters, 10(7): 211-214.

[7] Portilla, J. and Strela, V. (2003), Image denoising using scale mixtures of Gaussians in the wavelet domain. IEEE Transactions on Image Processing, 12(11): 1338-1351.

[8] Gopinathan S., Kokila R. and Thangavel P. (2015), Wavelet and FFT-based image denoising using non-linear filters. International Journal of Electrical and Computer Engineering, 5(5): 1018-1026.

[9] Jiang, X., Mahadevan, S. and Adeli, H. (2007), Bayesian wavelet packet denoising for structural system identification. Structural Control and Health Monitoring, 14(2): 333-356.

[10] Zhao, M. and Jia, X. (2017), A novel strategy for signal denoising using reweighted SVD and its applications to weak fault feature enhancement of rotating machinery. Mechanical Systems and Signal Processing, 94(September 2017): 129-147.

[11] Chatzi, E.N. and Fuggini, C. (2012), Structural identification of a super-tall tower by GPS and accelerometer data fusion using a multi-rate Kalman filter. In: Proceedings of the 3rd International Symposium on Life-Cycle Civil Engineering, Delft, Netherlands, 3-6 October 2012, 10: 144-151.

[12] Chatzi, E.N. and Fuggini, C. (2015), Online correction of drift in Structural Identification using artificial white noise observations and an Unscented Kalman filter. Smart Structures and Systems, 16(2): 296-328.

[13] Ravizza G., Ferrari R., Rizzi E. and Chatzi E.N. (2018), Effective heterogeneous data fusion procedure via Kalman filtering. Smart Structures and Systems, 22(5): 631-641.

[14] Pioldi, F., Ferrari, R. and Rizzi, E. (2015), Output-only modal dynamic identification of frames by a refined FDD algorithm at seismic input and high damping. Mechanical Systems and Signal Processing, 68-69(February 2016):265-291.

[15] Pioldi, F., Ferrari, R. and Rizzi, E. (2015), Earthquake structural modal estimates of multi-storey frames by a refined FDD algorithm. Journal of Vibration and Control, 23(13):2037-2063.

[16] Pioldi, F., Ferrari, R. and Rizzi, E. (2017), Seismic FDD modal identification and monitoring of building properties from real strong-motion structural response signals. Structural Control and Health Monitoring, 24(11):1-20.

[17] Moulin P. and Liu J. (1999), Analysis of multiresolution image denoising schemes using generalized Gaussian and complexity priors. IEEE Transactions on Information Theory, 45(3): 909-919. 
[18] Alfaouri M. and Daqrouq K. (2008), ECG signal denoising by wavelet transform thresholding. American Journal of Applied Sciences, 5(3): 276-281.

[19] Gradolewski D. and Redlarski G. (2013), The use of wavelet analysis to denoising of electrocardiography signal. In: XV International PhD Workshop OWD, 19-22 October 2013, pp. 456-461.

[20] Al-Qazzaz N.K., Ali S., Ahmad S.A., Islam M.S. and Arif M.I. (2015), Selection of mother wavelets thresholding methods in denoising multi-channel EEG signals during working memory task. Sensors, 15(11): 15-35.

[21] Sadooghi S.S. and Khadem E.K. (2016), A new performance evaluation scheme for jet engine vibration signal denoising. Mechanical Systems and Signal Processing, 76-77(2016): 201-212.

[22] Karthikeyan P., Murugappan M. and Yaacob S. (2012), ECG signal denoising using wavelet thresholding technique in human stress assessment. International Journal of Electrical Engineering and Information, 4(2): 306-319. 